\title{
Model-based adaptive phase I trial design of post-transplant decitabine maintenance in myelodysplastic syndrome
}

Seunghoon Han ${ }^{1,2}$, Yoo-Jin Kim³ ${ }^{3}$, Jongtae Lee ${ }^{1,2}$, Sangil Jeon ${ }^{1,2}$, Taegon Hong ${ }^{1,2}$, Gab-jin Park ${ }^{1,2}$, Jae-Ho Yoon ${ }^{3}$, Seung-Ah Yahng ${ }^{3}$, Seung-Hwan Shin ${ }^{3}$, Sung-Eun Lee ${ }^{3}$, Ki-Seong Eom ${ }^{3}$, Hee-Je Kim³ ${ }^{3}$, Chang-Ki Min ${ }^{3}$, Seok Lee ${ }^{3}$ and Dong-Seok Yim ${ }^{1,2^{*}}$

\begin{abstract}
Background: This report focuses on the adaptive phase I trial design aimed to find the clinically applicable dose for decitabine maintenance treatment after allogeneic hematopoietic stem cell transplantation in patients with higher-risk myelodysplastic syndrome and secondary acute myeloid leukemia.

Methods: The first cohort (three patients) was given the same initial daily dose of decitabine $\left(5 \mathrm{mg} / \mathrm{m}^{2} /\right.$ day, five consecutive days with 4-week intervals). In all cohorts, the doses for Cycles 2 to 4 were individualized using pharmacokinetic-pharmacodynamic modeling and simulations. The goal of dose individualization was to determine the maximum dose for each patient at which the occurrence of grade 4 (CTC-AE) toxicities for both platelet and neutrophil counts could be avoided. The initial doses for the following cohorts were also estimated with the data from the previous cohorts in the same manner.

Results: In all but one patient (14 out of 15), neutrophil count was the dose-limiting factor throughout the cycles. In cycles where doses were individualized, the median neutrophil nadir observed was 1100/ $\mathrm{mm}^{3}$ (grade 2) and grade 4 toxicity occurred in $5.1 \%$ of all cycles (while it occurred in $36.8 \%$ where doses were not individualized). The initial doses estimated for cohorts 2 to 5 were 4,5,5.5, and $5 \mathrm{mg} / \mathrm{m}^{2} /$ day, respectively. The median maintenance dose was $7 \mathrm{mg} / \mathrm{m}^{2} /$ day.
\end{abstract}

Conclusions: We determined the acceptable starting dose and individualized the maintenance dose for each patient, while minimizing the toxicity using the adaptive approach. Currently, $5 \mathrm{mg} / \mathrm{m}^{2} /$ day is considered to be the most appropriate starting dose for the regimen studied.

Trial registration: Clinicaltrials.gov NCT01277484

Keywords: Model-based drug development, Adaptive design, Myelodysplastic syndrome, Population pharmacokinetics-pharmacodynamics, Phase I clinical trial

\section{Background}

DNA methylation is the best-known epigenetic marker for cancer development [1]. In some hematologic malignancies including myelodysplastic syndrome (MDS), DNA methylation results not only in increased cell

\footnotetext{
*Correspondence: yimds@catholic.ac.kr

'Department of Pharmacology, College of Medicine, The Catholic University of Korea, 222 Banpo-Daero, Seochogu, Seoul, Republic of Korea

2PIPET (Pharmacometrics Institute for Practical Education and Training), 222

Banpo-Daero, Seochogu, Seoul, Republic of Korea

Full list of author information is available at the end of the article
}

proliferation but also in silencing of genes which regulate growth and differentiation [2]. Based upon those mechanisms, the use of a DNA hypomethylating agent (HMA) for hematologic malignancies has been expanded. Accordingly, clinical researches to optimize HMA therapy $[3,4]$ or to explore epigenetic mechanisms for new drug development have been widely performed $[5,6]$.

Decitabine (Dacogen ${ }^{\circ}, 5$-aza-2'-deoxycytidine) is a HMA that exerts its antitumor activity by inhibiting DNA methylation at low doses and by arresting DNA synthesis at high doses $[7,8]$. For several decades, decitabine has 
been one of the most intensely studied anticancer agents in the field of hematology due to its sophisticated development history [9-11], as well as its impressive clinical outcomes against many hematologic diseases [7, 9, 12-17]. For MDS, the approved indication for decitabine, numerous efforts have been made to optimize the dosing regimen according to patient characteristics, including the regimen evaluated in this study (five consecutive days of dosing with 4-week interval) [12, 18-22].

Recently, HMA maintenance therapy after allogeneic hematopoietic stem cell transplantation (allo-HSCT) has been suggested as a potentially attractive approach to minimize relapse and to improve graft survival [23-25]. Several studies on azacitidine (Vidaza ${ }^{\circ}, 5$-azacytidine) reported low toxicity, along with its potential to increase the number of hematopoietic stem cells [11, 26-29]; thus, similar approaches using decitabine were initiated [22]. In this context, we designed and performed a phase I study that aimed to find a clinically applicable dosage regimen for decitabine maintenance treatment after alloHSCT in patients with higher-risk MDS and secondary acute myeloid leukemia (AML).

Our study design incorporated two major considerations: (1) the purpose of the maintenance therapy was to maintain disease-free status in the patient while simultaneously preserving graft function, and (2) the dosage regimen should be determined using the smallest number of patients possible. Considering these aspects, without a confident estimation of the appropriate starting dose, traditional fixed-dose escalation schemes [30] were considered inappropriate for the following reasons: (1) fatal toxicity (e.g., graft failure) might occur in some subjects, (2) the study might need too many patients to find the optimal dose [31], and (3) dose differences between cohorts might be too large or small. Thus, we introduced an adaptive dose individualization design based upon pharmacokinetic (PK)-pharmacodynamic (PD) modeling for the neutropenia and thrombocytopenia caused by decitabine.

Dose individualization of anticancer drugs using PKPD modeling has been theoretically proposed using simulated data [32, 33]; however, our report is the first to implement dose individualization using PK-PD modeling in patients in a phase I clinical trial. We endeavored to titrate the appropriate dose for each patient, with the goal of identifying the highest possible dose that did not result in severe hematologic toxicities. We also anticipated that this approach would more quickly accomplish the study's objectives and avoid having to test several cohorts for the dose escalation. This report focuses on the study design, the PK-PD model development for hematologic toxicities caused by decitabine, and the usefulness of our adaptive approach as it applies to subject safety.

\section{Results}

\section{Patient characteristics}

Five patients with secondary AML evolving from MDS and 11 with MDS (9 males, 7 females) were enrolled (Table 1). All the patients received the myeloablative condition regimen and peripheral blood stem cells from the related $(n=6)$ or unrelated $(n=10)$ donors. The engraftment achievement of platelet and neutrophil counts was confirmed for all patients by an experienced hematologist upon enrollment. Graft-versus-host disease (GVHD) prophylaxis was calcineurin inhibitors (cyclosporine for related and tacrolimus for unrelated donors) plus short-course methotrexate. Antithymocyte globulin was given to all patients. Decitabine was administered at a median of 86 days (range, 56-90 days) after transplantation. At the time of decitabine treatment, acute ( $\leq$ overall grade 2 ) or chronic GVHD was observed in nine and one patients, respectively. The clinical features are given in Table 2.

\section{Patient disposition and dataset}

Patient dispositions are detailed in Fig. 1. In cohort 1, the third patient dropped out of the study without PD sampling; thus, we substituted with an additional patient, since PK-PD results from three patients were needed to obtain the initial dose for cohort 2. Fourteen patients completed all the study-related procedures until Cycle 4, and maintenance dose was determined for each patient at the end of Cycle 4 (Table 2).

For each subject, PK sampling was performed according to the protocol, and the average number of PD observations used in individual dose titration (IDT) was 5.76/cycle for both neutrophils and platelets. Among 58 treatment

Table 1 Patient demographics

\begin{tabular}{lllllll}
\hline Variables & Cohort & & & & \\
\cline { 2 - 5 } & 1 & 2 & 3 & 4 & 5 & Total \\
\hline Age (year) & $41.0 \pm 17.1$ & $57.0 \pm 12.1$ & $55.7 \pm 5.8$ & $55.3 \pm 11.7$ & $39.7 \pm 17.9$ & $49.2 \pm 14.4$ \\
Sex (male/female) & $3 / 1$ & $2 / 1$ & $0 / 3$ & $3 / 0$ & $1 / 2$ & $9 / 7$ \\
Height $(\mathrm{m})$ & $1.68 \pm 0.05$ & $1.65 \pm 0.10$ & $1.58 \pm 0.00$ & $1.71 \pm 0.05$ & $1.65 \pm 0.14$ & $1.65 \pm 0.08$ \\
Weight $(\mathrm{kg})$ & $55.4 \pm 5.5$ & $65.0 \pm 13.1$ & $52.1 \pm 1.8$ & $69.4 \pm 5.0$ & $60.3 \pm 8.6$ & $60.1 \pm 9.2$ \\
Body surface area $\left(\mathrm{m}^{2}\right)$ & $1.6 \pm 0.1$ & $1.7 \pm 0.2$ & $1.5 \pm 0.0$ & $1.8 \pm 0.1$ & $1.7 \pm 0.2$ & $1.7 \pm 0.2$ \\
\hline
\end{tabular}


Table 2 Patient characteristics and doses given in each subject, cohort, and cycle

\begin{tabular}{|c|c|c|c|c|c|c|c|c|c|c|}
\hline \multirow[t]{2}{*}{ Cohort } & \multirow{2}{*}{$\begin{array}{l}\text { Subject } \\
\text { number }\end{array}$} & \multirow{2}{*}{$\begin{array}{l}\text { Sex/ } \\
\text { age }\end{array}$} & \multirow{2}{*}{$\begin{array}{l}\text { WHO } \\
\text { diagnosis }\end{array}$} & \multirow[t]{2}{*}{ Donor } & \multicolumn{2}{|c|}{ GVHD grade ${ }^{a}$} & \multicolumn{4}{|c|}{ Cycle (mg/m²/day for 5 days) } \\
\hline & & & & & Acute & $\overline{\text { Chronic }}$ & 1 & 2 & 3 & 4 \\
\hline \multirow[t]{4}{*}{1} & 1 & $F / 19$ & RAEB-2 & MSD & 0 & 0 & $5^{\mathrm{b}, \mathrm{c}}$ & 1.5 & 1.5 & 1.5 \\
\hline & 2 & $M / 36$ & AML & MSD & 0 & 0 & $5^{\mathrm{b}}$ & 6 & 5.5 & 6 \\
\hline & 3 & $\mathrm{M} / 60$ & RAEB-2 & MUD & 1 & 0 & 5 & - & - & - \\
\hline & 4 & $M / 48$ & AML & MSD & 0 & 0 & $5^{\mathrm{b}, \mathrm{c}}$ & 1.5 & 2.5 & 3 \\
\hline \multirow[t]{3}{*}{2} & 5 & $M / 64$ & RAEB-2 & PMUD & 1 & 0 & $4^{b}$ & 4 & 5.5 & 7 \\
\hline & 6 & $F / 43$ & RAEB-2 & MSD & 0 & 0 & $4^{b}$ & 7 & 8 & 12 \\
\hline & 7 & $M / 64$ & AML & MSD & 0 & 0 & $4^{b}$ & 6 & 5.5 & 5.5 \\
\hline \multirow[t]{3}{*}{3} & 8 & $F / 51$ & RAEB-2 & MUD & 1 & 0 & $5^{b}$ & $7.5^{\mathrm{b}, \mathrm{c}}$ & $7.5^{c}$ & 7 \\
\hline & 9 & $F / 59$ & RAEB-1 & MUD & 2 & 0 & $5^{b, c}$ & 3.5 & 4 & 4.5 \\
\hline & 10 & $F / 36$ & RAEB-2 & MUD & 1 & 0 & $5^{b}$ & $6^{b}$ & 8.5 & 9 \\
\hline \multirow[t]{3}{*}{4} & 11 & $M / 64$ & RAEB-2 & MSD & 2 & 0 & $5.5^{b}$ & $2^{b}$ & - & - \\
\hline & 12 & $\mathrm{M} / 60$ & RAEB-2 & MSD & 0 & Mild & $5.5^{\mathrm{b}, \mathrm{c}}$ & $4.5^{b}$ & 7 & 8 \\
\hline & 13 & $M / 41$ & RAEB-2 & MSD & 0 & 0 & $5.5^{b, c}$ & 3 & 5 & 8 \\
\hline \multirow[t]{3}{*}{5} & 14 & M/49 & $\mathrm{AML}$ & MUD & 2 & 0 & $5^{b}$ & 1.5 & 2.5 & 3 \\
\hline & 15 & $\mathrm{~F} / 50$ & AML & MSD & 2 & 0 & $5^{b, c}$ & 4 & 6 & 9 \\
\hline & 16 & $F / 49$ & RAEB-2 & MSD & 2 & 0 & $5^{b}$ & 7.5 & 8 & $11^{\circ}$ \\
\hline
\end{tabular}

GVHD graft-versus-host disease, RAEB refractory anemia with excess blast, MSD matched sibling donor, PMUD partially matched unrelated donor, MUD matched unrelated donor

${ }^{a}$ Assessed at the time of decitabine initiation

${ }^{\mathrm{b}}$ Individual dose titration (IDT) by the PK-PD model was not applied

'The cycles where grade 4 toxicities occurred

cycles of 15 patients, the doses for Cycles 2 to 4 (a total of 39 cycles) were determined through PK-PD model-based adaptive dose individualization. Cycle 2 doses in four patients were clinically determined for the following reasons: no significant blood cell count decrease after cycle 1 (subjects 8 and 10) and not enough time for PK-PD modeling and IDT from sudden changes in visit schedules for Cycle 2 dosing (subjects 11 and 12). The actual dosing interval was $34.5 \pm 8.7$ days (mean $\pm \mathrm{SD}$ ).

\section{Estimated doses and safety outcomes}

In all but one patient (14 out of 15), the absolute neutrophil count (ANC) was the dose-limiting factor throughout all cycles. During the cycles in which IDT was performed, the median ANC nadir observed was 1100/ $\mathrm{mm}^{3}$ (range, $300 / \mathrm{mm}^{3}$ to $2680 / \mathrm{mm}^{3}$ ). The maintenance dose determined with four cycle data was higher than the initial doses in 10 out of the 15 patients. The initial doses (Cycle 1 doses) estimated by cohort dose estimation (CDE) were $4,5,5.5$, and $5 \mathrm{mg} / \mathrm{m}^{2} /$ day for cohorts $2,3,4$, and 5 , respectively. The median individual maintenance dose of decitabine was $7 \mathrm{mg} / \mathrm{m}^{2} /$ day (Table 2). Maintenance doses for the patients with Cycle 1 data inadequate for PK-PD modeling could be estimated using three cycle data (Cycles 2, 3, and 4) with acceptable model fits.
A total of nine dose-limiting toxicities (DLT, platelet count for one case and absolute neutrophil count for eight cases) were observed. Among these toxicities, seven cases occurred in non-IDT cycles (six in Cycle 1 and one in Cycle 2 with clinically determined doses). In the observed toxicities, $36.8 \%$ of the non-IDT cycles (7 out of 19 cycles) showed dose-limiting toxicities, which was an approximately seven times higher occurrence rate than that observed in the IDT cycles (5.1 \%, 2 out of 39 cycles).

\section{Overall mixed-effect PK-PD analysis}

A total of 95 PK observations and 622 PD observations (311 for ANC and 311 for platelet count, PC) were used in the overall mixed-effect PK-PD analysis. The one patient whose dose-limiting factor was PC was excluded from this analysis, whose disease entity was considered not to be similar to others, as she suffered from immune thrombocytopenia after transplantation and was managed with steroids. Among the data, $6.9 \%$ (4 out of 58 cycles) was obtained from the cycles where IDT was not applied.

A two-compartment model was found to best describe the PK data. The between-subject variability (BSV) for $C L$ (clearance from the central compartment) was the only random effect which could be estimated, except for the proportional residual error. The basic structure of the PD model was identical to that used for IDT and 


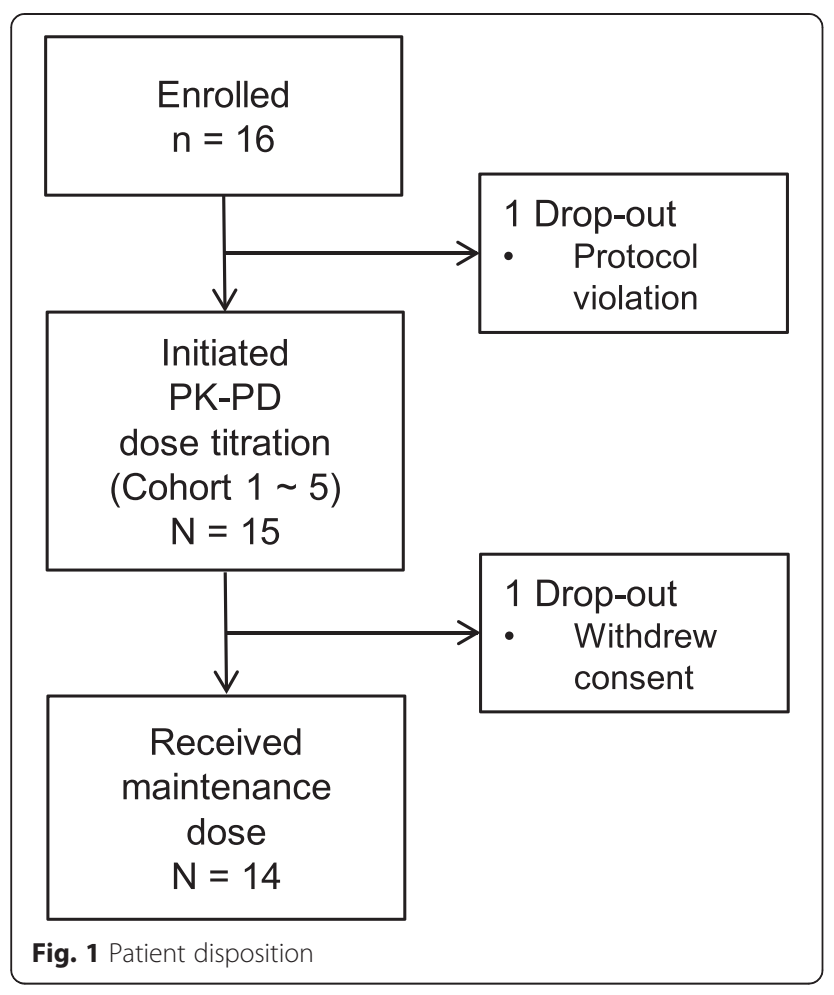

CDE for both PC and ANC (transit compartment model with feedback mechanism):

$$
\begin{aligned}
\frac{d A(1)}{d t}=k_{\mathrm{tr}} \cdot A(1) \cdot\left\{(1-\mathrm{SLOPE} \cdot C) \cdot(\mathrm{BASE} / A(5))^{\mathrm{GAMMA}}-1\right\} \\
\frac{d A(2)}{d t}=k_{\mathrm{tr}} \cdot(A(1)-A(2)) \\
\frac{d A(3)}{d t}=k_{\mathrm{tr}} \cdot(A(2)-A(3)) \\
\frac{d A(4)}{d t}=k_{\mathrm{tr}} \cdot(A(3)-A(4)) \\
\frac{d A(5)}{d t}=k_{\mathrm{tr}} \cdot(A(4)-A(5))
\end{aligned}
$$

where $A(N)$ is the cell count in the Nth compartment and $C$ is the plasma decitabine concentration. A detailed description for the parameters is presented in Table 3. $B A S E$ is a parameter indicating the level of cell count maintained at baseline or at the period without drug effect. For platelets, an asymptotic structure describing gradual cell count increase over cycles improved the model significantly, and thus the following structure substituted the simple BASE parameter:

$$
\mathrm{BASE}_{p}+\operatorname{IMP}\left(1-e^{-\mathrm{IMK} * \mathrm{TIME}}\right)
$$

where $I M P$ is the empirical value of the maximum PC recovery expected, $I M K$ is the rate constant for asymptotic PC recovery, and TIME is the time from the initiation of decitabine treatment.

No meaningful covariate was found in either the patient demographic or clinical variables. The parameter descriptions and estimates are given in Table 3. Simulated time courses of ANC changes, under the maintenance dosage of $5 \mathrm{mg} / \mathrm{m}^{2} /$ day for four treatment cycles, are presented in Fig. 2.

\section{Clinical course and non-hematological events}

During four cycles of the dose-finding phase of this study, one patient (subject 3) died of pneumonia (protocol violation) while the other two (subject 1 and subject 11) also suffered from pneumonia but fully recovered. One of the three cases developed decitabine-induced neutropenia (subject 11, withdrawn). Aggravation of existing acute or chronic GVHD was not observed, while chronic GVHD was diagnosed in two patients (one in mild and the other in moderate form). Herpes zoster was a complication in three patients.

\section{Discussion}

We succeeded in administering the maximum dose allowed for each patient, with minimized toxicity. The dose for each cycle was determined based upon the observed cell counts in the previous cycle(s) which are the ultimate outcome of patient characteristics and drug effect. Thus, the dose can be considered as a reflection of the vulnerability of the graft, the sensitivity to decitabine, and any possible drug interactions affecting cell counts. This method meant that using a large number of cohorts, as typically required in the traditional dose escalation scheme, could be avoided. Moreover, the doses of four patients were reduced from their initial doses because of their relatively vulnerable PD characteristics. The treatment of these patients might have been discontinued if a traditional, fixed-dose design had been used. Most importantly, our study design showed the significant advantage that all dose individualization steps were accomplished with a favorable toxicity profile, judging from the proportion of cycles that exhibited grade 4 toxicities. When IDT was applied, the proportion of cycles exhibiting grade 4 toxicities dropped to approximately one-seventh the level (36.8 versus $5.1 \%$ ) compared with the non-IDT cycles. Thus, model-based dose individualization can be a useful option in earlyphase clinical trial designs, in particular when the initial dose cannot be set with sufficient confidence.

The PK properties of decitabine in Korean patients obtained here are similar to those in previous studies. Liu et al. [34] and Cashen et al. [35] reported that the PK properties of decitabine could be well described with a two-compartment model. The distributional characteristics from these two studies could be indirectly compared using the maximum concentration $\left(C_{\max }\right)$ predicted upon the completion of decitabine infusion. From previous reports, the maximum concentration of decitabine was within the range of $60-70 \mathrm{ng} / \mathrm{mL}$, which was obtained 
Table 3 Final parameter estimates and bootstrap outcomes

\begin{tabular}{|c|c|c|c|c|c|c|}
\hline \multirow[t]{2}{*}{ Parameter } & \multirow[t]{2}{*}{ Unit } & \multirow[t]{2}{*}{ Description } & \multicolumn{2}{|c|}{ Population typical value } & \multicolumn{2}{|c|}{ Between-subject variability } \\
\hline & & & Estimate & Bootstrap median (95 \% Cl) & $\begin{array}{l}\text { Estimate } \\
\text { (as CV\%) }\end{array}$ & $\begin{array}{l}\text { Bootstrap } \\
\text { median }(95 \% \mathrm{Cl})\end{array}$ \\
\hline \multicolumn{7}{|c|}{$\begin{array}{l}\text { Pharmacokinetic } \\
\text { parameters }\end{array}$} \\
\hline$C L$ & $\mathrm{~L} / \mathrm{h} \cdot \mathrm{m}^{2}$ & Clearance & 87.8 & $88.3(72.2-108)$ & 21.4 & $20.5(13.0-26.8)$ \\
\hline$V_{c}$ & $\mathrm{~L} / \mathrm{m}^{2}$ & Volume of central compartment & 18.5 & $18.2(14.2-23.5)$ & $\mathrm{NE}$ & $\mathrm{NE}$ \\
\hline$V_{p}$ & $\mathrm{~L} / \mathrm{m}^{2}$ & Volume of peripheral compartment & 22.9 & $21.9(15.7-46.1)$ & NE & NE \\
\hline$Q$ & $\mathrm{~L} / \mathrm{h} \cdot \mathrm{m}^{2}$ & Intercompartmental clearance & 13.1 & $13.3(10.0-19.6)$ & $\mathrm{NE}$ & NE \\
\hline \multicolumn{7}{|c|}{$\begin{array}{l}\text { Pharmacodynamic } \\
\text { parameters for platelet }\end{array}$} \\
\hline$k_{\mathrm{tr}, \mathrm{P}}$ & $h^{-1}$ & $\begin{array}{l}\text { Rate constant of inter-compartmental } \\
\text { platelet movement }\end{array}$ & 0.0244 & $0.0246(0.0236-0.0254)$ & $\mathrm{NE}$ & NE \\
\hline$S L O P E_{P}$ & - & Drug effect on platelet count & 0.0656 & $0.0676(0.0539-0.0930)$ & 25.7 & $20.8(1.30-56.0)$ \\
\hline$B A S E_{P}$ & $/ \mathrm{mm}^{3}$ & Baseline platelet count & 49,200 & $53,700(36,100-95,800)$ & 105 & $78.3(38.3-110)$ \\
\hline$G A M M A_{p}$ & - & $\begin{array}{l}\text { Shape factor for platelet count } \\
\text { fluctuation }\end{array}$ & 0.304 & $0.299(0.264-0.325)$ & $\mathrm{NE}$ & NE \\
\hline IMP & & $\begin{array}{l}\text { Maximum degree of platelet count } \\
\text { recovery expected }\end{array}$ & 55000 & $58700(24200-98300)$ & 78.3 & $72.9(19.5-150)$ \\
\hline IMK & & $\begin{array}{l}\text { Rate constant for asymptotic platelet } \\
\text { count recovery }\end{array}$ & 0.000530 & $0.000513(0.000213-0.000691)$ & $\mathrm{NE}$ & NE \\
\hline \multicolumn{7}{|c|}{$\begin{array}{l}\text { Pharmacodynamic } \\
\text { parameters for neutrophil }\end{array}$} \\
\hline$k_{\mathrm{tr}, \mathrm{N}}$ & $h^{-1}$ & $\begin{array}{l}\text { Rate constant of inter-compartmental } \\
\text { neutrophil movement }\end{array}$ & 0.0132 & $0.0133(0.0120-0.0139)$ & $\mathrm{NE}$ & $\mathrm{NE}$ \\
\hline$S L O P E_{N}$ & - & Drug effect on neutrophil count & 0.263 & $0.237(0.114-0.363)$ & 57.5 & $58.1(25.8-119)$ \\
\hline$B A S E_{N}$ & $/ \mathrm{mm}^{3}$ & Baseline neutrophil count & 3240 & $3015(2110-4140)$ & 43.5 & $40.6(27.2-57.6)$ \\
\hline$G A M M A_{N}$ & - & $\begin{array}{l}\text { Shape factor for neutrophil count } \\
\text { fluctuation }\end{array}$ & 0.193 & $0.181(0.110-0.251)$ & 39.4 & $36.7(15.0-65.4)$ \\
\hline \multicolumn{7}{|c|}{ Residual error } \\
\hline$\sigma_{\mathrm{PK}}^{2}$ & - & $\begin{array}{l}\text { Variance of residual error } \\
\text { (proportional) for PK }\end{array}$ & 0.441 & $0.436(0.354-0.505)$ & - & - \\
\hline$\sigma_{\mathrm{PD}, \mathrm{P}}^{2}$ & & $\begin{array}{l}\text { Variance of residual error (additive) } \\
\text { for platelet count }\end{array}$ & 25,000 & $24,300(18,800-29,600)$ & - & - \\
\hline$\sigma_{P D, N}^{2}$ & & $\begin{array}{l}\text { Variance of residual error (additive) } \\
\text { for neutrophil count }\end{array}$ & 754 & $748(613-839)$ & - & - \\
\hline
\end{tabular}

Proportion of successful convergence: $78.8 \%$ for PK model, $78.0 \%$ for PD model $N E$ not estimated

approximately $1 \mathrm{~h}$ after initiation of infusion, when decitabine was administered at a rate of $5 \mathrm{mg} / \mathrm{m}^{2} / \mathrm{h}$ (3-h infusion of a $15 \mathrm{mg} / \mathrm{m}^{2}$ dose) $[35,36]$. This observation is consistent with our finding that the predicted $C_{\max }$ after 1-h infusion of $5 \mathrm{mg} / \mathrm{m}^{2}$ was $66.0 \mathrm{ng} / \mathrm{mL}$. In addition, the average terminal half-life was also similar $(0.31 \mathrm{~h}$ in this study); thus, the decitabine concentration is predicted to drop below $5 \%$ of $C_{\max }$ within $1.5-2 \mathrm{~h}$ after the completion of infusion.

The baseline cell count increase over cycles was modeled for platelet level. This was a consistent finding to the results from previous reports regarding the contribution of decitabine to cell proliferation [37-41]. For neutrophil counts, doses estimated by neutrophil count nadirs were gradually escalated over cycles until reaching the maintenance dose in ten patients while baseline cell count increase was not meaningful. Gradual deflation in the width of the prediction interval for ANC, resulting from improved precision of the model along with increased data points obtained throughout the cycles, seems to be one possible explanation. Dose escalation from this prediction interval deflation lowers the predicted median of course while maintaining the lower $25 \%$ prediction interval above $500 / \mathrm{mm}^{3}$ (grade 4 toxicity).

We also found it necessary to modify the interval between cycles that was initially planned as 4 weeks in this study. Although both $\mathrm{PC}$ and ANC were recovered to the baseline after decitabine dosing, our PK-PD model predicted that the time to nadir was 3.5 weeks and that 


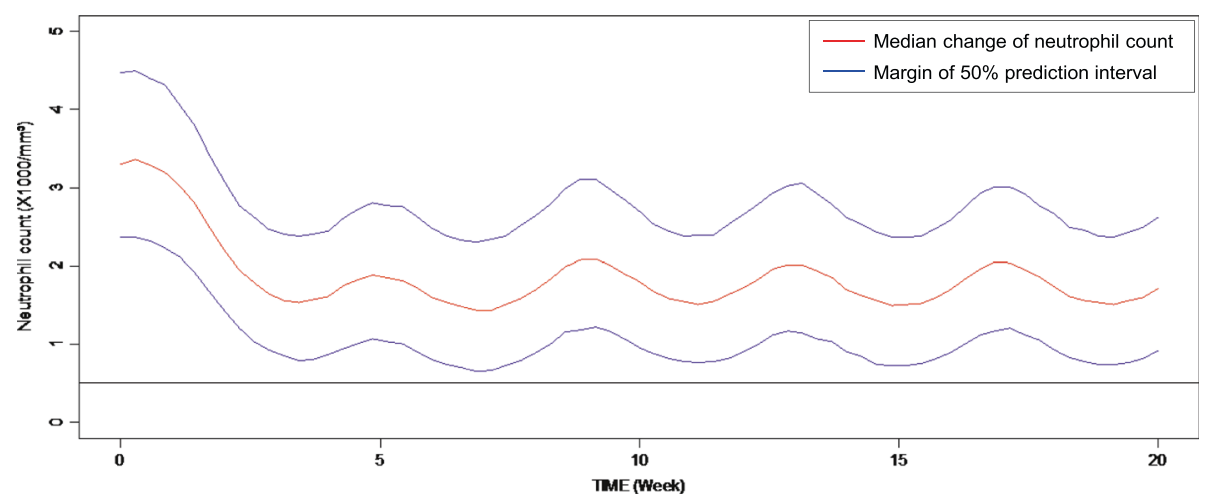

Fig. 2 Prediction of neutrophil count change when $5 \mathrm{mg} / \mathrm{m}^{2}$ dose is given for five consecutive days with 4-week interval. (From 1000 simulations using the final PK-PD model)

the time to recovery from the influence of the last dose (ANC $>1000 / \mathrm{mm}^{3}$ ) was approximately 5 weeks for the ANC. This prediction was consistent with the actual dosing interval practiced in this study (34.5 days on average). This finding implies that the 4-week interval may not be long enough to initiate the next cycle. Moreover, as illustrated in Fig. 2, the lowest value of ANC appears to be achieved in the second cycle (6-7 weeks after treatment initiation). Thus, the initial nadir of ANC within the first 4-week cycle should not be mistaken for the lowest ANC value throughout the cycles. This could also have been a reason for failure in dose determination if traditional fixed-dose escalation based on the first cycle nadir was recruited. To optimize the dosing regimen that may overcome this difficult property of decitabine, an initial loading dose may be considered before giving maintenance doses.

\section{Conclusions}

We exemplified the adaptive dose titration approach, based upon a quantitative exposure-toxicity model, in this study. This approach seemed most useful, since this method enabled rapid and precise dose individualization. The most appropriate initial dose was determined to be $5 \mathrm{mg} / \mathrm{m}^{2} /$ day for five consecutive days. Throughout the course of data analysis, issues such as extending between-cycle intervals and the use of loading doses were also raised. Cohort 6 is ongoing for exploration of the adequacy of the recommended starting dose, and additional report will be provided after completion of 12 cycles of treatment of all participants.

\section{Methods}

\section{Ethics, consent, and permission}

This study was designed and conducted in accordance with the principles of the Declaration of Helsinki and the good clinical practice guidelines of Korea. The independent institutional review board of Seoul St. Mary's
Hospital approved this study protocol before the initiation of any study-related procedure, and written informed consent was obtained from every subject. The registration number of this trial at "ClinicalTrials.gov" is NCT01277484.

\section{Patient eligibility}

Patients starting decitabine treatment on days 42-90 after allo-HSCT and meeting the following criteria were considered eligible: adult aged $\leq 65$; recipient of alloHSCT for higher-risk (intermediate 2 or high risk) MDS, as assessed by the International Prognostic Scoring System [42], and/or AML evolving from MDS; disease remission with appropriate recoveries of $\mathrm{PC}>30,000$ / $\mathrm{mm}^{3}$ and ANC $>1000 / \mathrm{mm}^{3}$, both of which were maintained for more than 7 days without any transfusions or growth factors; absence of grade III/IV acute GVHD; Eastern Cooperative Oncology Group (ECOG) performance status of 0 to 2; and no evidence of renal or hepatic impairment.

\section{Study design}

Patients were assigned to cohorts according to their order of enrollment. A cohort consisted of three patients to whom the same initial daily dose of decitabine (according to body surface area) was given. The initial dose for cohort 1 was $5 \mathrm{mg} / \mathrm{m}^{2} /$ day. The designated dose was infused intravenously over $60 \mathrm{~min}$ daily for five consecutive days in each cycle, and the cycle was repeated every 4 weeks up to Cycle 12. However, dosing was suspended if blood cell counts insufficiently recovered (PC $<30,000 / \mathrm{mm}^{3}$, ANC $<1000 / \mathrm{mm}^{3}$ ).

For cycles 2 to 4, the dose for each cycle was estimated using IDT according to PK-PD modeling and simulations based on blood cell count data accumulated until the time of dose estimation (just before administration). The maximum dose at which the occurrence of grade 4 hematologic toxicity (dose-limiting toxicity, $\mathrm{PC}<25,000$ / 
$\mathrm{mm}^{3}$ or ANC $<500 / \mathrm{mm}^{3}$ ) could be avoided at the lower limit of the $50 \%$ prediction interval (25th percentile), according to 500 simulations, was determined to be the dose for the next cycle. If the data from the previous cycle were not adequate for PK-PD modeling (e.g., no significant blood cell count decreases), the dose was determined based upon the hematologist's clinical decision [43]. Only the upper limit of the dose increment was pre-determined that the next cycle dose cannot exceed $150 \%$ of the previous dose. The dose determined at Cycle 4 for each individual was maintained thereafter.

The fixed initial dose for each cohort was also estimated using PK-PD modeling and simulations and was based on the observations from the previous cohorts $(\mathrm{CDE})$. For cohort 2, all of the data obtained before the initiation of treatment for the first patient in cohort 2 were used for the initial dose estimation; however, only Cycle 1 data from the previous cohorts were used for cohort 3,4 , and 5. A new cohort was not initiated before completion of the first cycle in the last patient of the previous cohort.

A schematic diagram of the overall study design is presented in Fig. 3.

\section{PK and PD samplings}

To determine plasma concentration measurements, seven whole-blood samples (10 $\mathrm{mL}$ each) were collected using EDTA tubes before dosing and then at 20, 40, 60, 90, 120, and $180 \mathrm{~min}$ after initiation of the first dose infusion of Cycle 1. The samples were immediately cooled in an ice bath and then centrifuged (3000 rpm, $4{ }^{\circ} \mathrm{C}$, for $10 \mathrm{~min}$ ) within $1 \mathrm{~h}$ from the last sampling time. After centrifugation, $4 \mathrm{~mL}$ of plasma from each sample was aliquoted into four microtubes $(1 \mathrm{~mL}$ each), and $10 \mu \mathrm{L}$ of $10 \mathrm{mg} / \mathrm{mL}$ tetrahydrouridine (THU) solution was added to each microtube. Microtubes were stored at -70 ${ }^{\circ} \mathrm{C}$ until plasma concentration assays.
As PD (toxicity) markers, PCs and ANCs were monitored at scheduled follow-up visits (weekly until Cycle 4 and biweekly thereafter). The procedures for obtaining PCs and ANCs followed the routine clinical practices for automated complete blood cell counts at Seoul St. Mary's Hospital.

\section{Plasma concentration measurements}

Plasma samples were analyzed using liquid chromatography coupled with tandem mass spectrometry (API 4000, ABSciex, Canada) based upon a previously reported method [34]. The lower limit of quantification (LLOQ) was $0.5 \mathrm{ng} / \mathrm{mL}$. The coefficients of correlation $\left(r^{2}\right)$ were greater than 0.9975 in the range of $0.5-100 \mathrm{ng} / \mathrm{mL}$ decitabine, as determined by weighted linear regression (1/concentration). The precision (\% coefficient of variation) and mean intraand inter-day accuracies were below $11.57 \%$ and 95.55$102 \%$, respectively.

\section{PK-PD modeling and simulation}

A mixed-effect analysis was performed using NONMEM (ver. 7.2, Icon Development Solution, Ellicott City, MD, USA). During the early phase of this study (e.g., IDT for cohort 1 and CDE for cohort 2), during which sufficient PD data to build a robust model were unavailable, we adopted the PD model proposed by Wallin et al. (2009) [33]. This model was used in conjunction with the onecompartment, first-order elimination PK model to build the initial PK-PD model. Therefore, only the values of the PK-PD parameters for each individual were estimated at this step. Then, as data accumulated, we performed additional modeling to find a better PK-PD model structure that optimally fits the data. Multi-compartment PK models, in addition to PD structures such as baseline cell count increase, were tested in the modeling process.

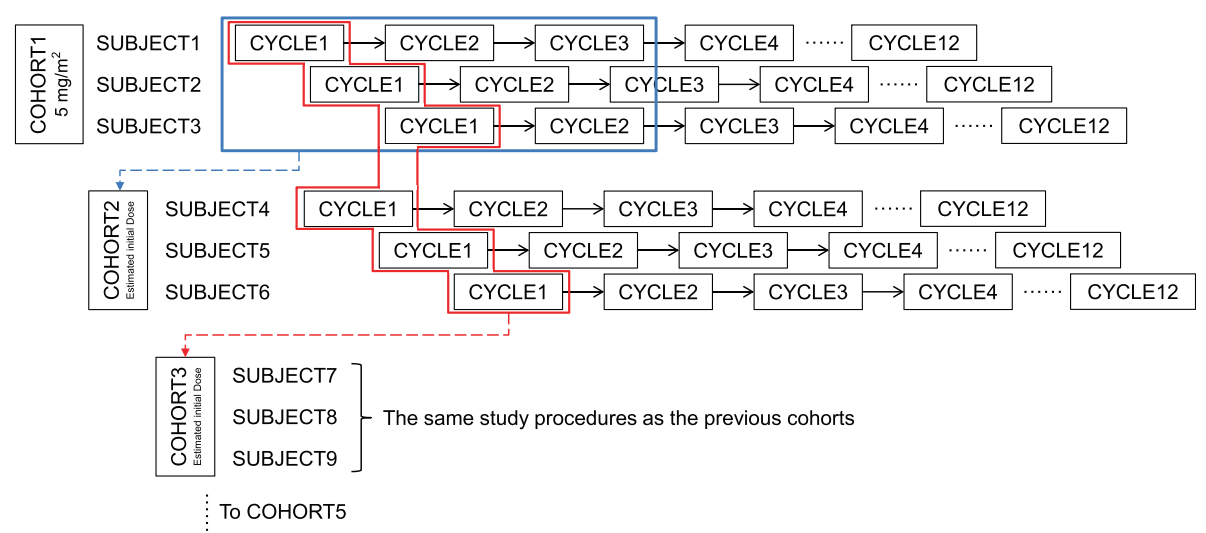

Fig. 3 Overall schema of the study design. Individual dose titration was performed for the next cycle based on the observations from the previous cycle (solid straight arrows). Cohort dose estimation was performed to determine initial doses (broken line arrows): (i) for cohort 2, using all data obtained from cohort 1 until the initiation of cohort 2; (ii) for cohorts 3-5, using only Cycle 1 data of previous subjects. The dose of Cycle 4 was maintained until the completion of decitabine treatment (Cycle 12) (dotted lines) 
Random effects were also taken into consideration. The structure to describe the residual error, which refers to the deviation of each observation from the value predicted by the PK-PD model, was initially applied to both IDT and CDE procedures as follows:

$$
\mathrm{DV}_{i j}=\operatorname{IPRED}_{i j} \cdot\left(1+\varepsilon_{\mathrm{prop}, i j}\right)+\varepsilon_{\mathrm{add}, i j}
$$

where $\mathrm{DV}_{i j}$ is the $j$ th measured concentration or blood cell count in the $i$ th individual, IPRED $i j$ is the modelpredicted value for the corresponding observation $\left(\mathrm{DV}_{i j}\right)$, and $\varepsilon_{\text {prop }, i j}$ and $\varepsilon_{\mathrm{add}, i j}$ are the residual variabilities with means of 0 and variances of $\sigma_{\text {prop }}^{2}$ and $\sigma_{\text {add }}^{2}$, respectively. For the CDE step, BSV $\left(\eta_{i}\right)$ of each PK and PD parameter was tested as follows:

$$
P_{i j}=T V P_{j} \cdot \exp \left(\eta_{i}\right)
$$

where $P_{i j}$ is the $j$ th model parameter in the $i$ th individual and $T V P_{j}$ is the typical value of the $j$ th model parameter. The BSV for each parameter was assumed to follow a normal distribution, with a mean of 0 and differing values of variance (described using the symbol $\omega_{i}^{2}$ ).

The first-order conditional estimation with interaction option (FOCE-I) method was used whenever applicable. Model adequacies were assessed based upon goodness-offit plots, likelihood ratio tests (LRT), and model stability measures (e.g., successful convergence, matrix singularity, and significant digits). Cutoff criteria incorporated a $p$ value of 0.05 (e.g., 3.84 for one parameter addition, 5.99 for two) for LRT to determine statistically significant improvements in the model.

Covariate analysis was performed for potential covariates, including demographic variables (sex, age, baseline body weight, and surface area) and clinical variables (mainly results from laboratory tests). After covariate screening via visual correlation check-ups and generalized additive modeling (GAM) procedures, the variables selected from the screening were tested as fixed effects for a certain PK-PD parameter, using LRT and decreases in $\mathrm{BSV}$ for the corresponding parameter.

\section{Competing interests}

An immediate family member of the author Yoo-Jin Kim has been employed, has had a leadership role, and has owned stock of the Janssen Pharmaceutical Companies of Johnson \& Johnson.

\section{Authors' contributions}

SH, Y-JK, JL, S-EL, C-KM, and D-SY wrote the manuscript. SH, Y-JK, J-HY, S-AY, S-EL, K-SE, H-JK, C-KM, SL, and D-SY designed the research. SH, Y-JK, JL, SJ, TH, G-JP, S-HS, S-EL, and D-SY performed the research. SH, Y-JK, JL, SJ, TH, and D-SY analyzed data. All authors read and approved the final manuscript.

\section{Acknowledgements}

This research was supported by the Janssen Pharmaceutical Companies of Johnson \& Johnson.

\section{Author details}

${ }^{1}$ Department of Pharmacology, College of Medicine, The Catholic University of Korea, 222 Banpo-Daero, Seochogu, Seoul, Republic of Korea. ${ }^{2}$ PIPET
(Pharmacometrics Institute for Practical Education and Training), 222 Banpo-Daero, Seochogu, Seoul, Republic of Korea. ${ }^{3}$ Catholic Blood and Marrow Transplantation Center, Seoul St. Mary's Hospital, The Catholic University of Korea, 222 Banpo-Daero, Seochogu, Seoul, Republic of Korea.

Received: 13 August 2015 Accepted: 28 September 2015 Published online: 23 October 2015

\section{References}

1. Esteller M. Epigenetics in cancer. N Engl J Med. 2008;358:1148-59.

2. Issa JP, Baylin SB, Herman JG. DNA methylation changes in hematologic malignancies: biologic and clinical implications. Leukemia. 1997;11:S7-11.

3. van der Helm LH, Scheepers ER, Veeger NJ, Daenen SM, Mulder AB, van den Berg $E$, et al. Azacitidine might be beneficial in a subgroup of older AML patients compared to intensive chemotherapy: a single centre retrospective study of 227 consecutive patients. J Hematol Oncol. 2013;6:29.

4. Pleyer $L$, Stauder R, Burgstaller S, Schreder M, Tinchon C, Pfeilstocker M, et al. Azacitidine in patients with WHO-defined AML - results of 155 patients from the Austrian Azacitidine Registry of the AGMT-Study Group. J Hematol Oncol. 2013;6:32.

5. Bachegowda L, Gligich O, Mantzaris I, Schinke C, Wyville D, Carrillo T, et al. Signal transduction inhibitors in treatment of myelodysplastic syndromes. J Hematol Oncol. 2013;6:50.

6. Hájková H, Fritz MH, Haškovec C, Schwarz J, Šálek C, Marková J, et al. CBFBMYH11 hypomethylation signature and PBX3 differential methylation revealed by targeted bisulfite sequencing in patients with acute myeloid leukemia. J Hematol Oncol. 2014;7:66.

7. Jabbour E, Issa JP, Garcia-Manero G, Kantarjian H. Evolution of decitabine development: accomplishments, ongoing investigations, and future strategies. Cancer. 2008;112:2341-51.

8. Santini V, Kantarjian HM, Issa JP. Changes in DNA methylation in neoplasia: pathophysiology and therapeutic implications. Ann Intern Med. 2001;134:573-86.

9. deVos D, van Overveld W. Decitabine: a historical review of the development of an epigenetic drug. Ann Hematol. 2001;84:S3-8.

10. Lübbert M. DNA methylation inhibitors in the treatment of leukemias, myelodysplastic syndromes and hemoglobinopathies: clinical results and possible mechanisms of action. Curr Top Microbiol Immunol. 2000;249:135-64.

11. Estey EH. Epigenetics in clinical practice: the examples of azacitidine and decitabine in myelodysplasia and acute myeloid leukemia. Leukemia. 2013;27:1803-12.

12. Kantarjian HM, Thomas XG, Dmoszynska A, Wierzbowska A, Mazur G, Mayer J, et al. Multicenter, randomized, open-label, phase III trial of decitabine versus patient choice, with physician advice, of either supportive care or low-dose cytarabine for the treatment of older patients with newly diagnosed acute myeloid leukemia. J ClinOncol. 2012;30:2670-7.

13. Issa JP, Gharibyan V, Cortes J, Jelinek J, Morris G, Verstovsek S, et al. Phase II study of low-dose decitabine in patients with chronic myelogenous leukemia resistant to imatinibmesylate. J ClinOncol. 2005;23:3948-56.

14. Oki Y, Kantarjian HM, Gharibyan V, Jones D, O'brien S, Verstovsek S, et al. Phase II study of low-dose decitabine in combination with imatinibmesylate in patients with accelerated or myeloid blastic phase of chronic myelogenous leukemia. Cancer. 2007;109:899-906.

15. Saunthararajah $Y$, Molokie R, Saraf S, Sidhwani S, Gowhari M, Vara S, et al. Clinical effectiveness of decitabine in severe sickle cell disease. $\mathrm{Br} \mathrm{J}$ Haematol. 2008;141:126-9.

16. Kantarjian H, Issa JP, Rosenfeld CS, Bennett JM, Albitar M, DiPersio J, et al. Decitabine improves patient outcomes in myelodysplastic syndromes: results of a phase III randomized study. Cancer. 2006;106:1794-803.

17. Santos FP, Kantarjian H, Garcia-Manero G, Issa JP, Ravandi F. Decitabine in the treatment of myelodysplastic syndromes. Expert Rev Anticancer Ther. 2010;10:9-22.

18. Wijermans PW, Lübbert M, Verhoef G, Klimek V, Bosly A. An epigenetic approach to the treatment of advanced MDS; the experience with the DNA demethylating agent 5-aza-2'-deoxycytidine (decitabine) in 177 patients. Ann Hematol. 2005;84:S9-17.

19. Lübbert M, Suciu S, Baila L, Rüter BH, Platzbecker U, Giagounidis A, et al. Low-dose decitabine versus best supportive care in elderly patients with intermediate- or high-risk myelodysplastic syndrome (MDS) ineligible for intensive chemotherapy: final results of the randomized phase III study of 
the European Organisation for Research and Treatment of Cancer Leukemia Group and the German MDS Study Group. J Clin Oncol. 2011;29:1987-96.

20. Kantarjian HM, O'Brien S, Huang X, Garcia-Manero G, Ravandi F, Cortes J, et al. Survival advantage with decitabine versus intensive chemotherapy in patients with higher risk myelodysplastic syndrome: comparison with historical experience. Cancer. 2007;109:1133-7.

21. Steensma DP, Baer MR, Slack JL, Buckstein R, Godley LA, Garcia-Manero G, et al. Multicenter study of decitabine administered daily for 5 days every 4 weeks to adults with myelodysplastic syndromes: the alternative dosing for outpatient treatment (ADOPT) trial. J Clin Oncol. 2009;27:3842-8.

22. Joeckel TE, Lübbert M. Clinical results with the DNA hypomethylating agent 5-aza-2'-deoxycytidine (decitabine) in patients with myelodysplastic syndromes: an update. Semin Hematol. 2012;49:330-41.

23. Goodyear O, Agathanggelou A, Novitzky-Basso I, Siddique S, McSkeane T, Ryan G, et al. Induction of a CD8+ T-cell response to the MAGE cancer testis antigen by combined treatment with azacitidine and sodium valproate in patients with acute myeloid leukemia and myelodysplasia. Blood. 2010;116:1908-18.

24. Choi J, Ritchey J, Prior JL, Holt M, Shannon WD, Deych E, et al. In vivo administration of hypomethylating agents mitigate graft-versus-host disease without sacrificing graft-versus-leukemia. Blood. 2010;116:129-39.

25. Schroeder T, Fröbel J, Cadeddu RP, Czibere A, Dienst A, Platzbecker U, et al. Salvage therapy with azacitidine increases regulatory $T$ cells in peripheral blood of patients with AML or MDS and early relapse after allogeneic blood stem cell transplantation. Leukemia. 2013;27:1910-3.

26. Jabbour E, Giralt S, Kantarjian H, Garcia-Manero G, Jagasia M, Kebriaei P, et al. Low-dose azacitidine after allogeneic stem cell transplantation for acute leukemia. Cancer. 2009;115:1899-905.

27. de Lima M, Giralt S, Thall PF, de Padua SL, Jones RB, Komanduri K, et al. Maintenance therapy with low-dose azacitidine after allogeneic hematopoietic stem cell transplantation for recurrent acute myelogenous leukemia or myelodysplastic syndrome: a dose and schedule finding study. Cancer. 2010;116:5420-31.

28. Schroeder T, Czibere A, Platzbecker U, Bug G, Uharek L, Luft T, et al. Azacitidine and donor lymphocyte infusions as first salvage therapy for relapse of AML or MDS after allogeneic stem cell transplantation. Leukemia. 2013;27:1229-35.

29. Platzbecker U, Wermke M, Radke J, Oelschlaegel U, Seltmann F, Kiani A, et al. Azacitidine for treatment of imminent relapse in MDS or AML patients after allogeneic HSCT: results of the RELAZA trial. Leukemia. 2012;26:381-9.

30. Storer BE. Design and analysis of phase I clinical trials. Biometrics. 1989;45:925-37.

31. Piantadosi S, Fisher JD, Grossman S. Practical implementation of a modified continual reassessment method for dose-finding trials. Cancer ChemotherPharmacol. 1998;41:429-36.

32. Wallin JE, Friberg LE, Karlsson MO. Model-based neutrophil-guided dose adaptation in chemotherapy: evaluation of predicted outcome with different types and amounts of information. Basic Clin Pharmacol Toxicol. 2010;106:234-42.

33. Wallin JE, Friberg LE, Karlsson MO. A tool for neutrophil guided dose adaptation in chemotherapy. Comput Methods Programs Biomed. 2009;93:283-91.

34. Liu Z, Marcucci G, Byrd JC, Grever M, Xiao J, Chan KK. Characterization of decomposition products and preclinical and low dose clinical pharmacokinetics of decitabine (5-aza-2'-deoxycytidine) by a new liquid chromatography/tandem mass spectrometry quantification method. Rapid Commun Mass Spectrom. 2006;20:1117-26.

35. Cashen AF, Shah AK, Todt L, Fisher N, DiPersio J. Pharmacokinetics of decitabine administered as a 3 -h infusion to patients with acute myeloid leukemia (AML) or myelodysplastic syndrome (MDS). Cancer Chemother Pharmacol. 2008;61:759-66

36. Karahoca M, Momparler RL. Pharmacokinetic and pharmacodynamic analysis of 5-aza-2'-deoxycytidine (decitabine) in the design of its dose-schedule for cancer therapy. Clin Epigenetics. 2013;5:3-18.

37. Milhem M, Mahmud N, Lavelle D, Araki H, DeSimone J, Saunthararajah Y, et al. Modification of hematopoietic stem cell fate by 5aza 2'deoxycytidine and trichostatin A. Blood. 2004;103:4102-10.

38. Young JC, Wu S, Hansteen G, Du C, Sambucetti L, Remiszewski S, et al. Inhibitors of histone deacetylases promote hematopoietic stem cell self-renewal. Cytotherapy. 2004;6:328-36.

39. Chung YS, Kim HJ, Kim TM, Hong SH, Kwon KR, An S, et al. Undifferentiated hematopoietic cells are characterized by a genome-wide undermethylation dip around the transcription start site and a hierarchical epigenetic plasticity. Blood. 2009;114:4968-78.

40. Suzuki M, Harashima A, Okochi A, Yamamoto M, Nakamura S, Motoda R, et al. 5-Azacytidine supports the long-term repopulating activity of cord blood CD34(+) cells. Am J Hematol. 2004;77:313-5.

41. Negrotto S, Ng KP, Jankowska AM, Bodo J, Gopalan B, Guinta K, et al. CpG methylation patterns and decitabine treatment response in acute myeloid leukemia cells and normal hematopoietic precursors. Leukemia. 2012;26:244-54

42. Greenberg P, Cox C, LeBeau MM, Fenaux P, Morel P, Sanz G, et al, International scoring system for evaluating prognosis in myelodysplastic syndromes. Blood. 1997;89:2079-88.

43. Kantarjian H, Oki Y, Garcia-Manero G, Huang X, O'Brien S, Cortes J, et al. Results of a randomized of 3 schedules of low-dose decitabine in higher-risk myelodysplastic syndrome and chronic myelomonocytic leukemia. Blood. 2007;109:52-7.

\section{Submit your next manuscript to BioMed Central and take full advantage of:}

- Convenient online submission

- Thorough peer review

- No space constraints or color figure charges

- Immediate publication on acceptance

- Inclusion in PubMed, CAS, Scopus and Google Scholar

- Research which is freely available for redistribution 